\title{
Instantaneous Heart Rate Detection using Smart Phones Built-in Camera
}

\author{
Han $\mathrm{Li}^{1,}$ a , Hai Zhao ${ }^{1, b}$ \\ ${ }^{1}$ School of Computer Science \& Engineering Northeastern University \\ Heping District,Shenyang,110819, China \\ a lih_neu@163.com, b'zhaoh@mail.neu.edu.cn
}

Keywords: Instantaneous Heart Rate; Phase Detector ;Pulse Wave; Camera detect; Android Smart Phone;

\begin{abstract}
The limited smart phone battery and computational resources demand efficient and low-power consumption algorithms for the instantaneous heart rate detection using phone built-in camera. A phase detector based method is proposed to improve the performance of heart rate detection software using built-in camera. The pulse wave detected by the camera is multiplied by a sine wave reference signal and then processed by a low pass filter to extract phase. The instantaneous heart rate is calculated based on the phase difference between every two adjacent cycles. Experimental results on an Android smart phone indicated that there is no obvious difference of instantaneous heart rate between the new method and the ECG method. Compared with derivative based and filter bank based algorithm the new algorithm consume less phone resource and obtain instantaneous heart rate more quickly.
\end{abstract}

\section{Introduction}

Smart phones today have become a necessity for life with their diverse functionalities such as navigation, social networking and multimedia facilities.

Health monitoring mode is also expanded by the development of the phone processors, cameras and built-in sensors such as accelerometer and light-sensor [1]. Heart rate, breathing capacity, blood pressure etc have been reported measured on the smart phone[1-4]. We focus on the instantaneous heart rate measurement on an Android smart phone. Numerical study reveal that instantaneous heart rate can be used to assess cardiac functions[5-6]. Instantaneous heart rate is the reciprocal value of each ECG cycle. It is the reaction of heart rate speed and also the heart rate variability (HRV). Reduced HRV has been shown to be a predictor of mortality after myocardial infarction[7].

Most software in the smart phone application market can only detect average heart rate in 10 or 15 seconds. Because a threshold calculation based on a period of measurement is always necessary when using derivative based algorithms[8]. While other methods such as filter bank[9] or wavelet method[10]will be greatly affected on real time. A phase detector based algorithm is presented for the estimation of instantaneous heart rate from the pulse wave sensed by the phone camera.

\section{Method}

\subsection{Algorithm design}

To detect heart rate by a smart phone, the user should cover the phone camera by his fingertip. The fingertip blood color changes which represent the cardiac ejection circle can be detected by the camera image sensor. The outcome of the image senor is a video composed of many frame. Every frame grayscale varies according to the blood volume in the fingertip. When the blood waveform caused by the heartbeat flow to the capillaries, the blood oxygen increase and the blood become dark[10]. To detect instantaneous heart rate in real time, the algorithm needs to be simply enough to be completed in one heart beat e.g. 1-2 seconds. 
The phase detector which in general is a phase comparision component in a phase locked loop. It is used to detect phase difference between input signal and a feedback signal. Thus the instant frequency of the input signal can be obtained[11].

Phase detector can be implemented based on multipliers. The raw PPG signal is high-pass filtered to remove the baseline using a second-order Butterworth filter with a cut-off frequency at 0.5 $\mathrm{Hz}$.Assume that the heartbeat frequency components of the input signal is:

$$
\mathrm{u}_{\mathrm{i}}(\mathrm{t})=\mathrm{U}_{1} \sin \left[\omega_{\mathrm{i}} \mathrm{t}+\theta_{\mathrm{i}}(\mathrm{t})\right]
$$

The filtered signal is then routed into two separate multipliers with sine and cosine reference respectively. Two reference signal has the same amplitude $U 2$ and frequency $\omega r$ with only $\pi / 2$ phase difference. The frequency $\omega r$ is set to the estimated detector frequency of the previous iteration. Assume that the cosine reference signal is:

$$
\mathrm{u}_{\mathrm{r}}(\mathrm{t})=\mathrm{U}_{2} \cos \left[\omega_{\mathrm{r}} \mathrm{t}+\theta_{\mathrm{r}}(\mathrm{t})\right]
$$

A new signal is created as formula (3) shown through the multiplication of formula(1) and formula(2).

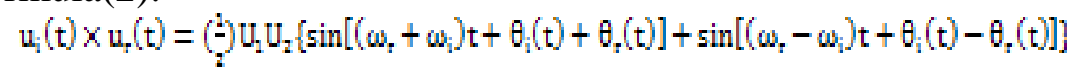

High frequency components can be removed through a low pass filter and thus the output is: $K_{d} \sin \Delta \theta$ and the other output is $K_{d} \cos \Delta \theta$.

$(2) \mathrm{U}_{2} \mathrm{U}_{2} \sin \left[\theta_{i}(\mathrm{t})-\theta_{x}(\mathrm{t})\right]=\mathrm{K}_{\mathrm{d}} \sin \Delta \theta$

where $\mathrm{K}_{\mathrm{d}}=(1 / 2) \mathrm{U}_{1} \mathrm{U}_{2}$.All other frequency components will also be shifted but they will not go through the low-pass filter.

$$
\omega_{\mathrm{r}}^{t}=\omega_{\mathrm{i}}^{\mathrm{t}-1}-\Delta \theta \quad \text { (5) }
$$

In order to eliminate the effect of amplitude, the tangent value is obtained based on $\mathrm{K}_{\mathrm{d}} \sin \Delta \theta / \mathrm{K}_{\mathrm{d}} \cos \Delta \theta$. Phase difference ${ }^{\Delta \theta}$ for heart rate in real time can be calculated using the inverse trigonometric function. The calculated instantaneous heart rate also serves as the reference signal frequency of the incoming period. Pulse wave process is shown in figure 1.

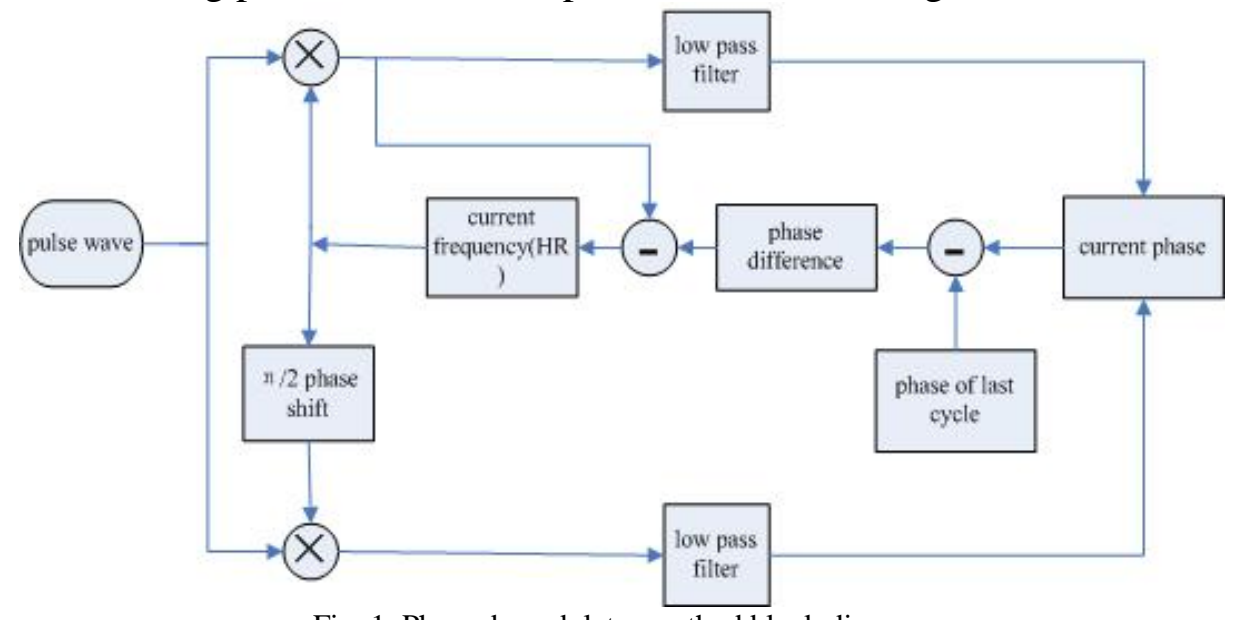

Fig. 1. Phase demodulator method block diagram

\subsection{Detection of pulse wave}

Android application is developed using eclipse (Helios ServiceRelease2) and android-SDK development. The finger blood change is collected and recorded by the phone camera. The testers need to put fingers close to the camera when measuring.

The reference signal frequency should be the same with camera sampling frequency. In general human's heart rate is $1-2 \mathrm{~Hz}$, so setting the camera sampling rate at 20 frames per second can meet the requirements. Green light intensity information is extracted because of the good absorption for hemoglobin. So the pixels in green channels of RGB are averaged separately for each frame.

In the Android application, Class "PreviewCallback" is used to control the camera Activity. The real time preview frame video can be obtained from parameter "data" of the method "public void onPreviewFrame(byte[] data, Camera camera)" in Class "PreviewCallback". 


\subsection{Lowpass filter realizaion}

We choose FIR filters for low pass filtering in fig.2 for the following reasons: (1) FIR filters can be constructed to have a strictly linear phase response, so that no phase distortion is introduced into the signal by the filter. The phase responses of IIR filters are nonlinear; (2) the effects of using a limited number of bits to implement filters such as round-off noise and coefficient quantization errors are much less severe in FIR than in IIR.

Because the low pass filtering and the phase calculation are dependent on the start time of each circle, a segmentation algorithm is proposed. As shown in figure3, the signal segmentation time is the last heart rate circle. The calculation start time is the last circle finish time adding the time corresponding to the compensation phase. The compensation phase is phase difference between the measured signal and reference signal of the last circle. In order to obtain the minimum value between the segmentation and the new heart beating circle, the segmentation time is constantly adjusted according to phase compensation. So the calculation segmentation time is not continuous.

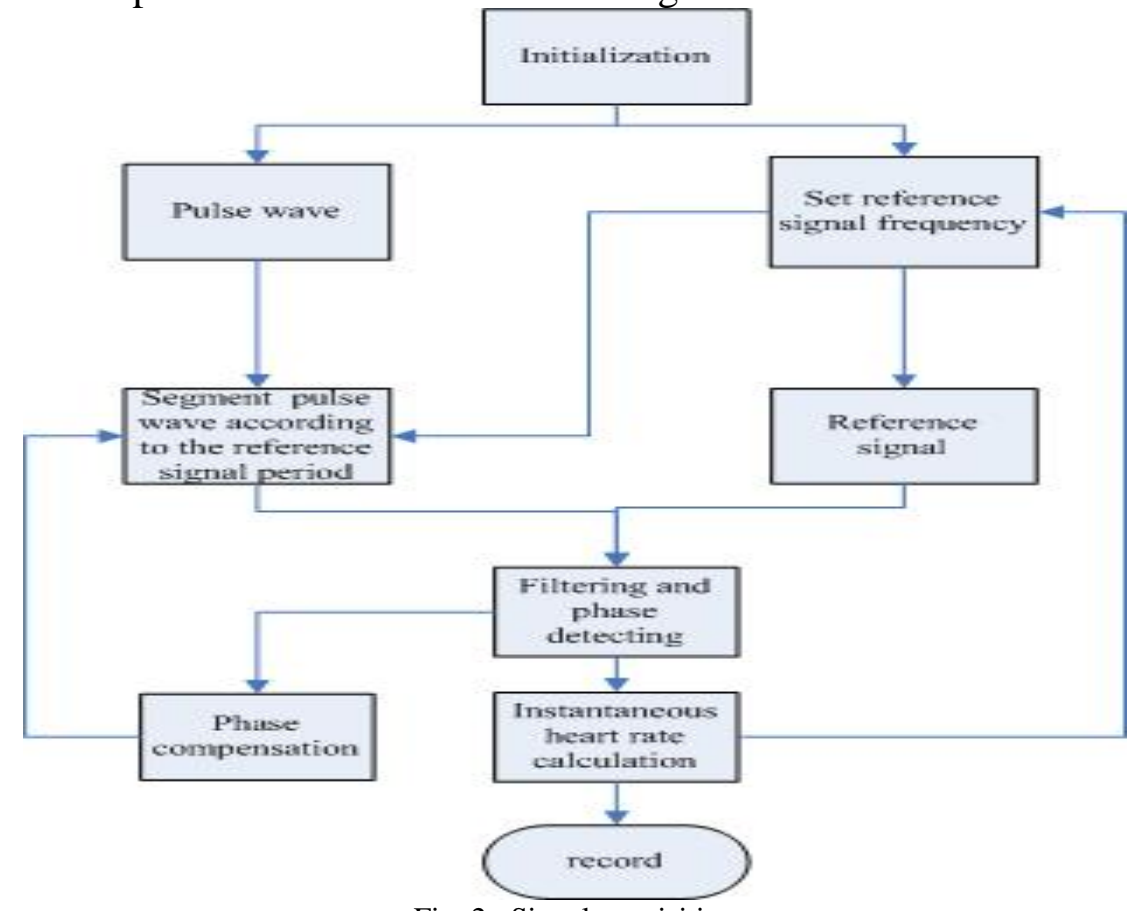

Fig. 2. Signal acquisition

\section{Experiment}

\subsection{Comparision algorithms}

\subsubsection{Derivative-based algorithms}

It is necessary to set a reasonable threshold based on a period of time before measuring the heart rate using derivative-based algorithms. The algorithm is based on the usage of the characteristic steep slope of the pulse wave.

Suppose S1 is the pulse wave signal after filtering and denoising. S2and S3are the first and second differential signals of S1 with thresholds respectively. The purpose of the second difference is to eliminate the effect of dicrotism.S3 is shown as equation(6).

$$
\left\{\begin{array}{c}
\mathrm{S}_{\mathrm{a}}(\mathrm{i})=\mathrm{S}_{2}(\mathrm{i}) \quad \mathrm{S}_{2}(\mathrm{i})<H \\
\mathrm{~S}_{\mathrm{a}}(\mathrm{i})=0 \quad \mathrm{~S}_{2}(\mathrm{i}) \geq \mathrm{H}
\end{array}\right.
$$

Where $\mathrm{H}$ is normally selected $\mathrm{as}^{\mathrm{H}}=\frac{\mathrm{z}}{\mathrm{N}} \sum_{\mathrm{i}=1}^{\mathrm{N}} \mathrm{s}_{2}$ (i). $\mathrm{N}$ is the number of sample points. In order to select the effective threshold $\mathrm{N}$ should be large enough to include several heart beats. That's why it takes almost 10 s to complete the first calculation.

\subsubsection{Filter bank}

Filter bank method is a method of extracting instantaneous heart rate in frequency domain. Filter bank is an array of band-pass filters that separates the input signal into multiple components. Each 
component carries a specific frequency sub-band of the input signal. Among the multiple components, the component with highest energy was selected and the frequency sub-band it carried was the instantaneous heart rate at $\mathrm{n}$-th second. The filter bank range is set from $0.5 \mathrm{~Hz} \sim 4 \mathrm{~Hz}$ with $0.05 \mathrm{~Hz}$ increase. A fixed 1-second window size of localized source signals are chosen as the inputs of filter bank to ensure that the most possible heart rate is estimated.

\subsection{Result}

\subsection{1 delay}

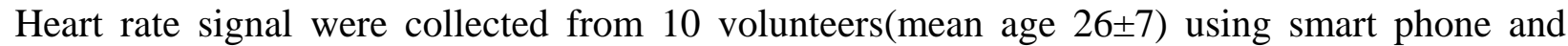
three-lead ECG at the same time for two minutes(right hand holding the phone with forefinger coving the camera and the two hands and right foot connecting to the ECG electrodes). The volunteers need to do exercise before the test for one minute in order to make heart rate change. Instantaneous heart rate measurements were recorded in the phone. ECG analog signal was processed by an ATM128 single chip computer to transmit to the PC for further analysis. The latency is defined as the average time difference between each R peak of ECG and the time of instantaneous heart rate calculation result on the phone. Figure3 indicates each heart beat average latency of three algorithms with different sampling frequency.

Higher resolution and frame sampling frequency will represent the blood change more accurately. However this will also lead to higher consumption of system resources. Resolution is decided by the phone camera and frame sampling frequency can be changed by software. So the resolution is fixed at $1280 * 720$ by the Samsung NOTE2 and we change different frame sampling frequency to design a reasonable test.

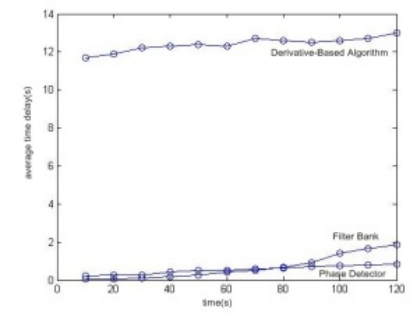

a)

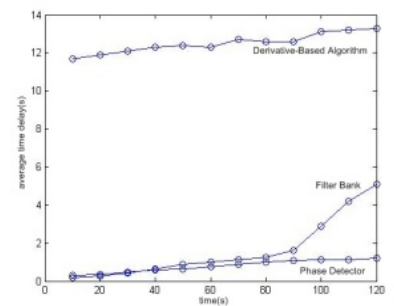

b)

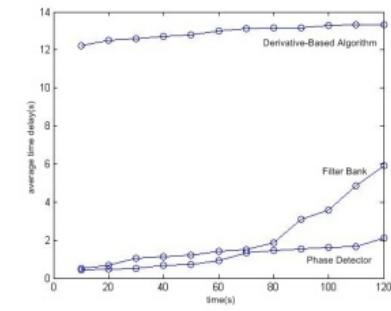

c)

Fig. 3.Average latency of the three method with different sampling frequency at a)10fps b)20fps and c)25fps

As shown in Figure.4 the delay time of the three algorithms increase gradually according to the frame sampling frequency respectively. Because of the need to train for a period of time to generate a threshold, there is fixed delay for the derivative-based algorithm.

Both the phase detector method and the filter bank method can complete instantaneous heart rate in time within 60s in the first chart. However, the average delay time of the latter method increase obviously as time goes on. The detector method also has increasing trend, but the delay time increase less compared to the filter bank method.

\subsubsection{Accuracy}

The sampling frequency is set at 20fps to test the accuracy of the three algorithms. To determine if adequate agreement existed across the phase method and ECG method, Bland-Altman ratio method is used to compare the accuracy[12]. The basic idea of Bland-Altman method is to calculate the consistency limits of the two kinds of measurement results and plot it. As shown in the Figure 5, the horizontal axis represents the average value of each heart beat measured by the two methods and the vertical represents the ratio of the two measured value. The upper and lower lines represent the limits of the differences between the two methods (the so-called 95\% limits of agreement). It can be seen from the chart the method proposed has good consistency with the ECG method with pearson correlation coefficient 0.94 and normalized root mean square $2.25 \%$. 


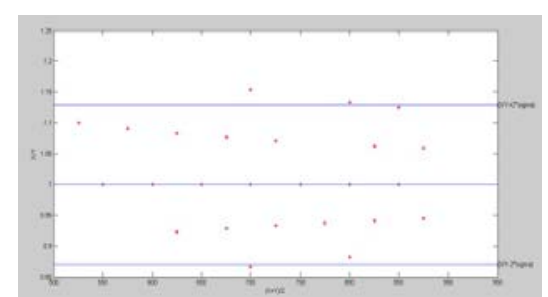

Fig. 4. Bland-Altman diagram of phase detector and ECG on instantaneous heart rate measurement

\subsubsection{Discussion}

360 mobile phone guardian is used to test memory use. As the chart shows the bank filter method consumes the most memory and the derivative-based algorithm consumes the least. Although the signal segmentation is required for all the three methods, the derivative-based algorithm needs only subtraction.

Table 1. Comparison of the three methods

\begin{tabular}{|c|c|c|c|}
\hline $\begin{array}{l}\text { Items/Algorith } \\
\text { m }\end{array}$ & $\begin{array}{l}\text { Derivative-ba } \\
\text { sed }\end{array}$ & Phase detector & Filter bank \\
\hline RMSE & $3.4 \%$ & $2.3 \%$ & $1.8 \%$ \\
\hline Delay & initialization & little delay & $\begin{array}{c}\text { Beat to beat } \\
\text { delay }\end{array}$ \\
\hline $\begin{array}{c}\text { Memory } \\
\text { consumption }\end{array}$ & $12.5 \mathrm{MB}$ & 32.6MB & $68.5 \mathrm{MB}$ \\
\hline
\end{tabular}

Phase detector based method can measure the instantaneous heart rate accurately with normalized root mean square 2.25\% (ECG as reference). It is worse than the bank filter method $1.8 \%$ but better than the 3.4\%. Accurate rate will dropped without phase compensation.

We conclude that the new method will meet the needs of daily heart rate measurement. However there are some deficiencies. In theory, the algorithm can detect arrhythmia, but no experiment was carried out in clinical trials. All the tests are performed still to reduce the impact of baseline drift and noise. Removing noise increasing the system resource consumption which may has an effect on the instantaneous heart rate calculation.

\section{Conclusions}

A novel approaches to computes instantaneous heart rate based on a phase detector algorithm were proposed. The computed HR was accurate with respect to the reference HR computed from ECG and compared favorably with other algorithms in terms of accuracy, delay and memory use. This makes the algorithms suitable to process PPG signals in real time on a smart phone. We intend to test the algorithm on smart phone during various movement-free tasks in the further work.

\section{ACKNOWLEDGMENT}

This work was supported by China National Natural Science Foundation Grant No.61101121.

\section{References}

[1]. V. Chandrasekaran, R. Dant, and S. Jonnada, Cuffless differential blood pressure estimation using smart phones, IEEE Transactions on Biomedical Engineering, vol. 60, no.2,pp. 1080-1089, 2013.

[2]. H.Li and H.Zhao, Systolic blood pressure estimation using Android smart phones, 2013 6rd International Conference on Biomedical Engineering and Informatics, Hangzhou, China, pp.260-264,2013.

[3]. M.Gregoski, M. Mueller and A.Vertegel et.al,Development and validation of a smartphone heart rate acquisition application for health promotion and wellness telehealth applications. International Journal of Telemedicine and Applications,vol.41,no.1,pp.1-7,2012. 
[4]. E.Larson,M.Goel and G.Boriello et.al,SpiroSmart: using a microphone to measure lung function on a mobile phone. Proceedings of the 2012 ACM Conference on Ubiquitous Computing, Newyork,USA,pp.280-289, 2012.

[5]. JL.JR.Bigger, JL.Fleiss and RC.Steinman et al, Frequency domain measures of heart period variability and mortality after myocardial infarction, Circulation, vol.85, no.1, pp.164-171, 1992.

[6]. RE.Kleiger, JP.Miller and JT. Bigger et al ,Decreased heart rate variability and its association with increased mortality after acute myocardial infarction, Am J Cardiol,vol.59,no.4, pp.256-262,1987.

[7]. SZ.Abildstrom ,BT.Jensen and E.Agner et al, Heart rate versus heart rate variability in risk prediction after myocardial infarction, Journal of Cardiovascular Electrophysiology,vol.14,no.2, pp.168-73,2003.

[8]. B.Kohler, C.Hennig, R.Orglmeister, The principles of software QRS detection. IEEE Engineering in Medicine and Biology Magazine,vol.21,no.1,pp.42-57,2002.

[9].Y.Yu, Heart rate estimation from facial images using filter bank. 2014 6th International Symposium on Communications, Control and Signal Processing, Athens, Greece,pp.69 - 72, 2014.

[10]. W.Szi,C.Hsiao,HL.Chanb, A real-time QRS detection method based on moving-averaging incorporating with wavelet denoising. Computer Methods and Programs in Biomedicine,vol.82, no.3,pp.187-195,2006.

[11]. Liu Shing-Hong, Chang Kang-Ming, Fu Tsu-Hsun. Heart rate extraction from photoplethysmogram on fuzzy logic discriminator.Engineering Applications of Artificial Intelligence, vol.23,no.6,pp.968-977,2010.

[12]. K.Dewittel, C.Fierensl and D.Stöckl et al. Application of the Bland-Altman plot for interpretation of method- comparison studies: a critical investigation of its practice[J].Clinical Chemistry, vol.48,no.5,pp.799-801 2002. 